\title{
Spin Waves Excitations of Co/Pt Multilayers
}

\author{
W. Zhou \\ Key Laboratory of Materials Physics, Institute of Solid State Physics, Chinese Academy of Sciences, Hefei 230031, China \\ Correspondence should be addressed to W. Zhou, weizhou@theory.issp.ac.cn
}

Received 25 July 2012; Accepted 25 September 2012

Academic Editor: Weichang Hao

Copyright ( $) 2012$ W. Zhou. This is an open access article distributed under the Creative Commons Attribution License, which permits unrestricted use, distribution, and reproduction in any medium, provided the original work is properly cited.

The present work investigated interlayer couplings of $[\operatorname{Co}(20 \AA) / \operatorname{Pt}(30 \AA)]_{5}, \quad[\operatorname{Co}(4 \AA) / \operatorname{Pt}(7 \AA)]_{30}$, and $[\operatorname{Co}(4 \AA) / \operatorname{Pt}(9 \AA)]_{30}$ multilayers with strong perpendicular magnetic anisotropy (PMA). Brillouin light scattering measurements were utilized to obtain spin waves of these samples with in-plane external magnetic fields. Interlayer couplings were found to be very sensitive to Pt thickness change from $7 \AA$ to $9 \AA$, which implies that Pt atoms were more difficult to be polarized to provide interlayer coupling between Co layers than in the perpendicular external magnetic field situation. When Pt layer is $30 \AA$, the observed single spin wave can confirm the disappearance of interlayer coupling even when Co layer thickness is $20 \AA$.

\section{Introduction}

Since the first observation of spin waves in 1946 by Griffiths [1], with wide applications of laser in light scattering experiments and the invention of multipass Febry Pérot interferometer by Sandercock in 1970 [2], Brillouin scattering became an important tool in the field of magnetism to detect different kinds of magnetic excitations in various systems, including bulk materials, thin films, and nanostructures. Magnetic thin film systems are of particular importance due to their rich magnetism physics and practical applications in magnetic recording industry; it includes systems of single magnetic films, double magnetic films, and multiple layered magnetic films composed by ferromagnetic (FM), antiferromagnetic (AFM), and nonmagnetic (NM) films of different thickness and layer arrangements, among which FM/NM multilayers gained a long-term interest in the last decade. Many theoretical and experimental works were devoted to this field in understanding its magnetic behaviors especially for the perpendicular magnetic anisotropy thin films [3-7] through different approaches like neutron scattering, ferromagnetic resonance, magneto-optical Kerr microscopy, and Brillouin scattering. Generally, external magnetic fields were applied perpendicular to the sample surface in PMA samples in various experimental methods mentioned above. However, in this work, we are going to apply magnetic fields inplane to three strong PMA samples $[\mathrm{Co}(20 \AA) / \mathrm{Pt}(30 \AA)]_{5}$,
$[\mathrm{Co}(4 \AA) / \operatorname{Pt}(7 \AA)]_{30}$, and $[\operatorname{Co}(4 \AA) / \operatorname{Pt}(9 \AA)]_{30}$ then use Brillouin scattering to detect spin waves, from which magnetic coupling behaviors can be deduced and compared with experiments with magnetic fields perpendicular to the sample surface. Due to the weak in-plane magnetic anisotropy, three strong PMA samples will have different spin waves when Co and Pt thickness changed thus providing another approach to understand magnetic coupling of this system.

\section{Experimental Procedure}

Ferromagnetic multilayers $[\mathrm{Co}(t 1) / \mathrm{Pt}(t 2)]_{N}$ with $t 1$ and t2 as thickness of corresponding Co and Pt layers with $N$ total periods were fabricated using a magnetron sputtering system of ultrahigh vacuum with working pressure of at least $10^{-9}$ torr. Three samples used in the present work are $[\mathrm{Co}(20 \AA) / \operatorname{Pt}(30 \AA)]_{5},[\mathrm{Co}(4 \AA) / \operatorname{Pt}(7 \AA)]_{30}$, and $[\mathrm{Co}(4 \AA) / \operatorname{Pt}(9 \AA)]_{30}$. All of them were fabricated on the Si substrate with native oxide and $100 \AA$ buffer layer of $\mathrm{Pt}$, after Co and Pt layers of designed thickness were deposited; $30 \AA \mathrm{Pt}$ was deposited as the capering layer. All samples were found to have perpendicular magnetic anisotropy using a Lake Shore vibrating sample magnetometer (VSM) in [8], where more details of sample fabrication and calibration can be found.

Brillouin light scattering experiments were carried out using a tandem six-pass Febry Pérot interferometer. 
A single p-polarized $514.5 \mathrm{~nm}$ laser was used as the excitation source with an output power of $50 \mathrm{~mW}$, focusing on a spot of approximately $50 \mu \mathrm{m}$ in diameter. A water cooling electromagnet was used to provide magnetic fields up to $10 \mathrm{KOe}$ in the direction parallel to the sample surface. The standard backscattering geometry was utilized in the whole experimental process, where the incident laser has different angles of $30^{\circ}, 45^{\circ}$, and $60^{\circ}$ with respect to the normal direction of sample surface. Scattered light from spin waves was analyzed by the software Ghost to get Stoke and Antistoke spin waves at different external magnetic fields.

\section{Results and Discussion}

Figure 1 shows Brillouin spectra of spin waves of sample $[\mathrm{Co}(20 \AA) / \mathrm{Pt}(30 \AA)]_{5}$ with laser incident angle of $60^{\circ}$, where the external magnetic field is parallel to the sample surface up to $8.0 \mathrm{KOe}$; the experiment geometry is shown in the inset of Figure 2. Spin wave frequencies increase with external magnetic fields almost linearly from approximately $10 \mathrm{GHz}$ at $1.0 \mathrm{KOe}$ to $30 \mathrm{GHz}$ at $8.0 \mathrm{KOe}$. In Figure 2, it shows a linear fit of 10 measured data points up to $10.4 \mathrm{KOe}$; the linear fitting function $y=6.86+3.02 * x$ has very small stand errors of intercept and slope as 0.536 and 0.086 ; the adjusted coefficient of determination (adj. $R$-square) is also a small number as 0.993. Thus, we believe that the observed spin wave energies increase linearly with the external magnetic fields $H$ rather than in the form of square root of $H$, which is a typical behavior of spin waves whose wave vector $q$ is parallel rather than perpendicular to the magnetization $M$. Besides spin waves, there are two weak peaks around $4 \mathrm{GHz}$ and $9 \mathrm{GHz}$ at high magnetic fields of 7.0 KOe and 8.0 KOe, which show no changes with magnetic fields; they were confirmed as phonons from Brillouin scattering measurements without magnetic fields. Similar phonons were also observed for the other two samples $[\mathrm{Co}(4 \AA) / \operatorname{Pt}(7 \AA)]_{30}$ in Figure 4 and $[\operatorname{Co}(4 \AA) / \operatorname{Pt}(9 \AA)]_{30}$ in Figure 5.

The incident angle dependence of spin waves frequency was checked by changing laser incident angle $\theta$ to the normal of sample surface from $60^{\circ}$ to $30^{\circ}$ and $45^{\circ}$ at different external magnetic fields. When the magnetic field was switched to the inverse direction, it was denoted as a negative field relative to the previous field in Figure 3. There are two observations from spectra in Figure 3. The first is that the spin waves at different incident angles $\theta$ have the same frequencies as those of $60^{\circ}$ plotted in Figures 1 and 2 within experimental error, which demonstrates independence of spin wave frequency with respect to the incident angle $\theta$. To confirm such a conclusion, we extended free spectra range to $100 \mathrm{GHz}$ and found no other spin waves. Thus, the single observed spin wave of sample $[\mathrm{Co}(20 \AA) / \operatorname{Pt}(30 \AA)]_{5}$ shows no surface spin wave behavior, whose frequency is incident angle dependent in the form of cosine. The second observation is that the left side of spectra (Stoke side) is weaker than the right side (Antistoke side) for negative magnetic fields; the situation was totally inversed when magnetic fields are positive. The same phenomena can also be observed in $\theta=60^{\circ}$ in Figure 1, where the magnetic fields are positive

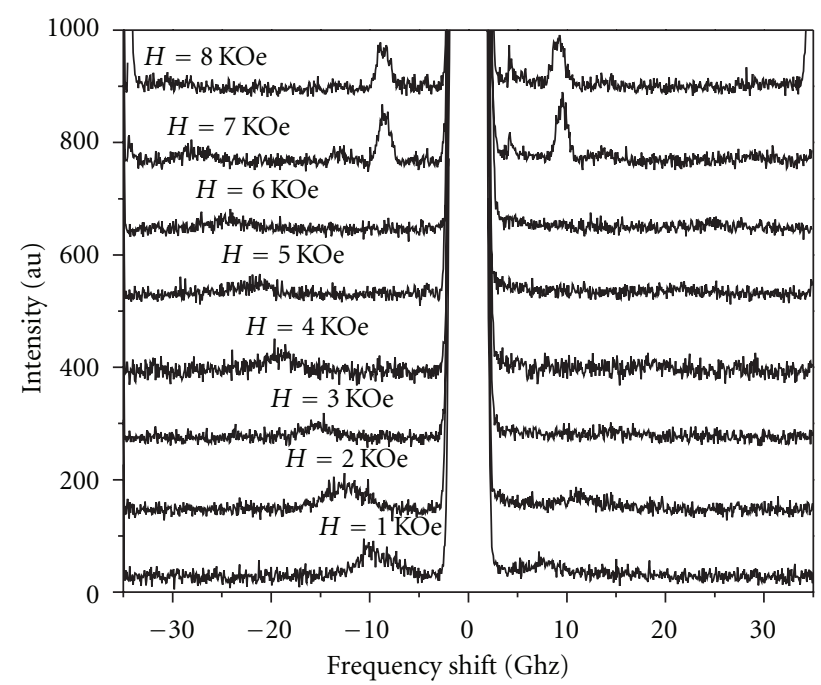

FIgUre 1: Spin wave Brillouin spectra of sputtered [Co $(20 \AA) /$ $\operatorname{Pt}(30 \AA)]_{5}$ sample with incident angle $\theta$ of $60^{\circ}$ and magnetic fields parallel to the sample surface up to $8.0 \mathrm{KOe}$.

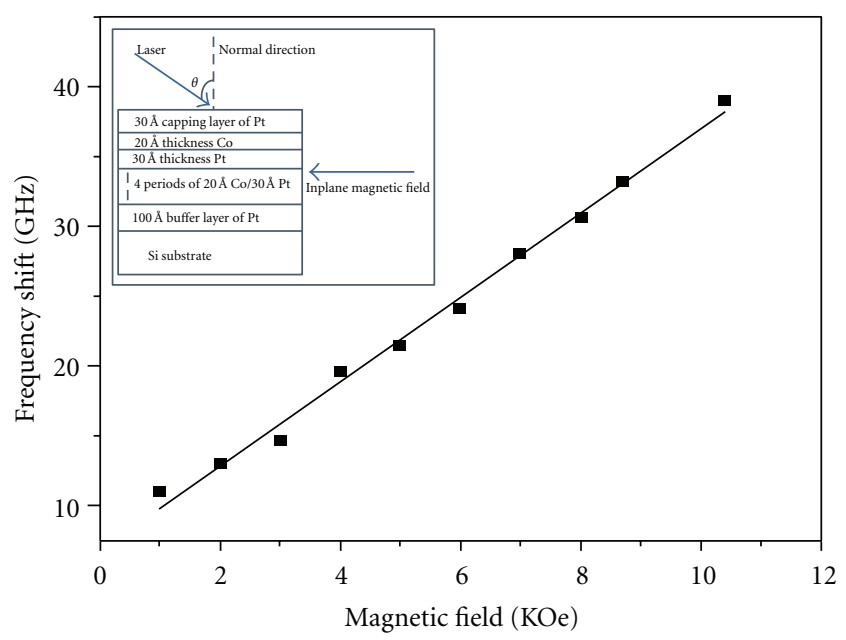

Figure 2: Magnetic field dependence of the spin wave frequencies of $[\mathrm{Co}(20 \AA) / \mathrm{Pt}(30 \AA)]_{5}$ sample with incident angle $\theta$ as $60^{\circ}$. The solid line is the linear fit of 10 measured data points. The inset shows the experiment geometry.

with Stoke side always stronger than Antistoke side. This is totally different from the normal acoustic excitations; their Stoke and Antistoke sides are of roughly the same intensity except for a temperature-dependent factor, which is a very small difference at room temperature compared with magnetic excitations intensity differences we observed. The explanation is due to the lack of time reversal symmetry for Stoke and Antistoke processes; spin wave intensity is proportional to the square of $G M \pm K$ as calculated in [9], where $M$ is the magnetization component and $K$ and $G$ are imaginary and real tensor part of dielectric constant fluctuations. \pm are for Stoke and Antistoke intensities, which are the origin of the observed spin wave intensity asymmetry. 


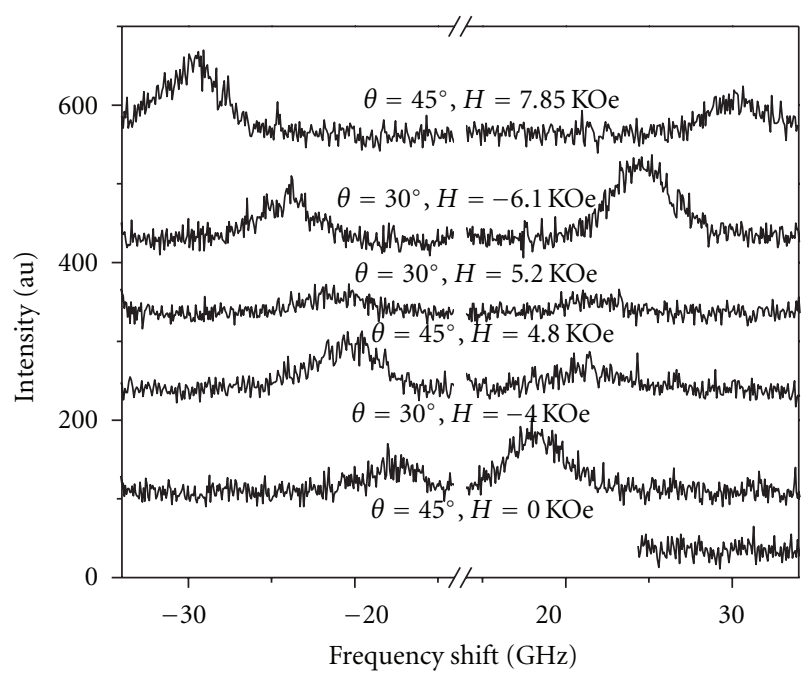

Figure 3: Spin wave Brillouin spectra of sputtered [Co $(20 \AA) /$ $\operatorname{Pt}(30 \AA)]_{5}$ sample with incident angle $\theta$ as $30^{\circ}$ and $45^{\circ}$ at different magnetic fields parallel to the sample surface. Negative and positive fields have opposite directions.

Brillouin spectra of the other two samples $[\operatorname{Co}(4 \AA) /$ $\operatorname{Pt}(7 \AA)]_{30}$ and $[\mathrm{Co}(4 \AA) / \operatorname{Pt}(9 \AA)]_{30}$ were presented in Figures 4 and 5. They both have $120 \AA$ Co layers, comparable with the $100 \AA$ Co layer of $[\mathrm{Co}(20 \AA) / \mathrm{Pt}(30 \AA)]_{5}$ sample. All three samples have strong perpendicular magnetic anisotropy with inplane magnetization much weaker from VSM measurements in [8]. In Figure 4, two acoustic phonons at low frequencies can be observed; the one with larger intensity is at $7.2 \mathrm{GHz}$ and the weaker one at $11.2 \mathrm{GHz}$; they have similar positions as phonons of sample $[\mathrm{Co}(4 \AA) / \mathrm{Pt}(9 \AA)]_{30}$, which are $6.6 \mathrm{GHz}$ for the stronger phonon and $10.2 \mathrm{GHz}$ for the weaker phonon separately. There are clearly two spin waves at $23.1 \mathrm{GHz}$ and $15.2 \mathrm{GHz}$ of sample $[\mathrm{Co}(4 \AA) / \mathrm{Pt}(7 \AA)]_{30}$ at magnetic fields of $\pm 6.5 \mathrm{KOe}$, whose Stoke and Antistoke relative intensities are different in the same manner as the spectra of $[\mathrm{Co}(20 \AA) / \mathrm{Pt}(30 \AA)]_{5}$ sample in Figures 1 and 3.

By comparing the spectra of $\pm 6.5 \mathrm{KOe}$ of $[\mathrm{Co}(4 \AA) /$ $\operatorname{Pt}(7 \AA)]_{30}$ sample in Figure 4 and that of $8.7 \mathrm{KOe}$ of $[\mathrm{Co}(4 \AA) / \operatorname{Pt}(9 \AA)]_{30}$ sample in Figure 5, one can find that spin waves of the former are stronger than those of the second sample. This can be attributed to their different Pt thickness, which is also their only difference. Because of the weak in-plane magnetic anisotropy, the external in-plane magnetic fields can only excite weak spin waves in thin $4 \AA$ Co layers compared with $20 \AA$ Co layers in the sample $[\mathrm{Co}(20 \AA) / \operatorname{Pt}(30 \AA)]_{5}$, where stronger spin waves can be obtained in Figure 3 . We thus understand that signal-to-noise ratio of spin waves is determined by the thickness of Co layer thickness. Another interesting observation is that the spin waves species are different between sample $[\mathrm{Co}(20 \AA) / \mathrm{Pt}(30 \AA)]_{5}$ and two later layered samples of 30 layers. By changing free spectra range to more than $100 \mathrm{GHz}$, we found only one spin wave for the sample $[\mathrm{Co}(20 \AA) / \mathrm{Pt}(30 \AA)]_{5}$ and two spin waves for samples $[\mathrm{Co}(4 \AA) / \operatorname{Pt}(7 \AA)]_{30}$ and $[\mathrm{Co}(4 \AA) / \operatorname{Pt}(9 \AA)]_{30}$.

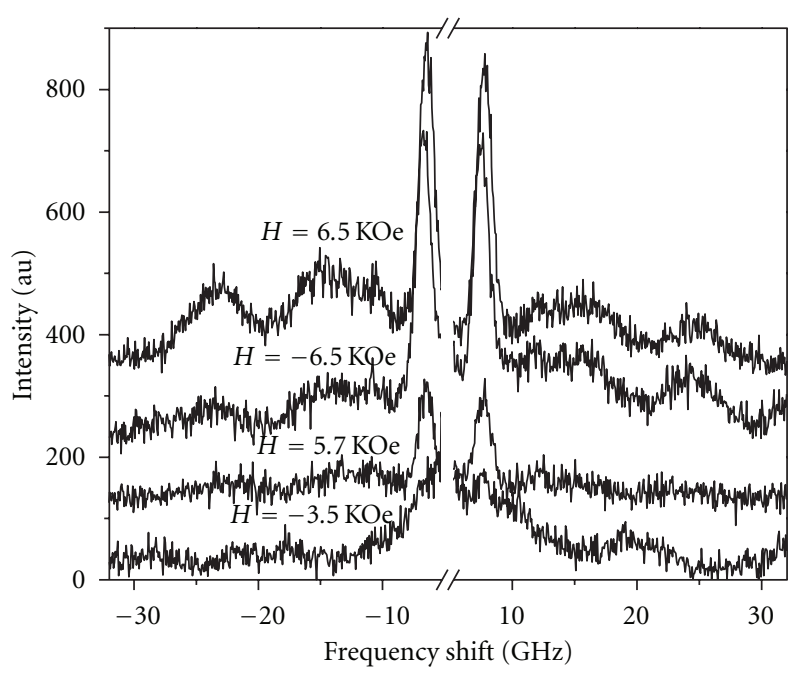

FIGURE 4: Spin wave Brillouin spectra of sputtered [Co $(4 \AA) /$ $\operatorname{Pt}(7 \AA)]_{30}$ sample with incident angle $\theta$ as $60^{\circ}$ at different magnetic fields parallel to the sample surface. Negative and positive fields have opposite directions.

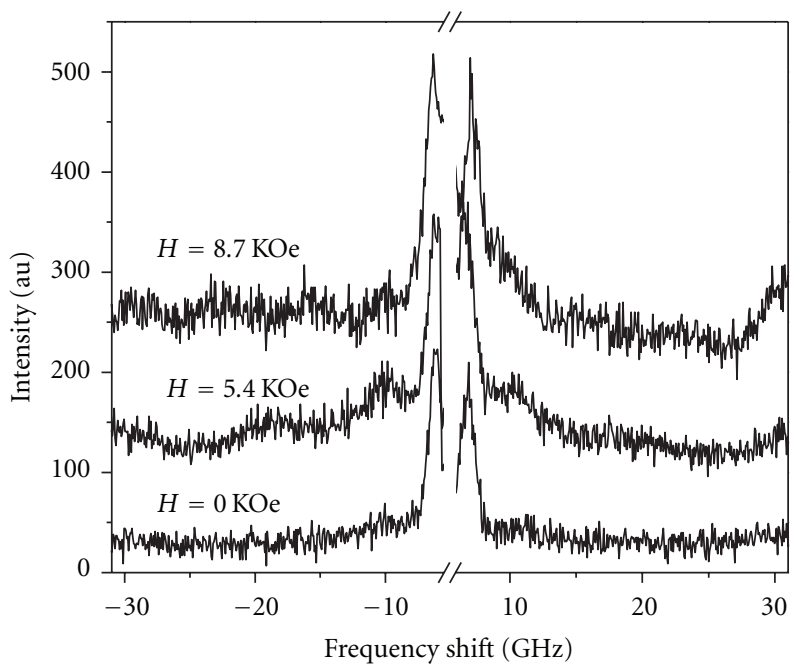

Figure 5: Spin wave Brillouin spectra of sputtered [Co $(4 \AA) /$ $\operatorname{Pt}(9 \AA)]_{30}$ sample with incident angle $\theta$ as $60^{\circ}$ at different magnetic fields parallel to the sample surface.

Also, their spin wave frequency behaviors are different under external magnetic fields. Besides the linear increasing of spin wave frequency with magnetic fields of sample $[\mathrm{Co}(20 \AA) / \operatorname{Pt}(30 \AA)]_{5}$ in Figure 2, spin wave frequency of the other two samples shifts clearly in a different manner, although due to the limited signal-to-noise ratio, not all spin waves of the two samples $[\operatorname{Co}(4 \AA) / \operatorname{Pt}(7 \AA)]_{30}$ and $[\mathrm{Co}(4 \AA) / \mathrm{Pt}(9 \AA)]_{30}$ can be clearly resolved. This difference can be attributed to the different interlayer couplings of ferromagnetic Co layers. In $[10,11]$, magneto-optical Kerr microscopy was used to investigate interlayer couplings of same batch of $[\mathrm{Co}(4 \AA) / \mathrm{Pt}(t)]_{N}$ multilayers with strong perpendicular magnetic anisotropy. When the Pt thickness is less than $11 \AA$, Co layers are strongly coupled. 
Although in our study, magnetic fields are parallel to the sample surface, the induced magnetization should still be coupled, which can be used to explain the observed two spin waves and their intensity decreasing when Pt layers changed from $7 \AA$ to $9 \AA$. So we conclude that the in-plane magnetization is much weaker to polarize $\mathrm{Pt}$ atoms to provide interlayer coupling of Co layers as in the perpendicular cases; thus, the spin waves are very weak and only observable at high magnetic fields, such as \pm 6.5 KOe spectra in Figure 4 . When Pt layers changed from $7 \AA$ to $9 \AA$, magnetic field up to $8.7 \mathrm{KOe}$ is still not enough to get clear spin wave signals as before. On the contrary, when Pt is of $30 \AA$ thickness, Kerr imaging can confirm that the interlayer couplings are quite small, which can explain the observed one spin wave in $[\mathrm{Co}(20 \AA) / \operatorname{Pt}(30 \AA)]_{5}$ sample, with almost no couplings between Co layers. Also, $20 \AA$ Co thickness layer can provide strong spin wave signals as in Figure 2.

\section{Conclusions}

In conclusion, interlayer coupling of three $\mathrm{Co} / \mathrm{Pt}$ multilayers has been investigated by Brillouin scattering with in-plane magnetic fields. We find one spin wave of $[\mathrm{Co}(20 \AA) /$ $\operatorname{Pt}(30 \AA)]_{5}$ sample; its frequency increases linearly with magnetic fields and independently of incident angles, which implies that Co interlayer coupling is negligibly small; two spin waves of $[\mathrm{Co}(4 \AA) / \operatorname{Pt}(7 \AA)]_{30}$ and $[\mathrm{Co}(4 \AA) / \operatorname{Pt}(9 \AA)]_{30}$ are also found, and their frequency behaviors are different from the first one, which demonstrates the existence of Co interlayer coupling and the decreasing of this coupling with increasing Pt thickness. Although we used in-plane magnetic fields to investigate perpendicular magnetic anisotropy samples, the behaviors of spin waves were found to be more sensitive to the Pt thickness change correspondingly, thus providing another approach to understand the multilayered magnetic thin film systems.

\section{Acknowledgments}

The author acknowledges Dr. F. Y. Yang, from The Ohio State University, for providing the multilayer samples, and Dr. R. Sooryakumar and X. P. Xie, from The Ohio State University, for helping in Brillouin scattering experiments and discussions. Support from the National Science Foundation of China (NSF no. 11204315) is gratefully acknowledged.

\section{References}

[1] J. H. E. Griffiths, "Anomalous high-frequency resistance of ferromagnetic metals," Nature, vol. 158, pp. 670-671, 1946.

[2] J. R. Sandercock, "Brillouin scattering study of SbSI using a double-passed, stabilised scanning interferometer," Optics Communications, vol. 2, pp. 73-76, 1970.

[3] M. Buchmeier, H. Dassow, D. E. Bürgler, and C. M. Schneider, "Intensity of Brillouin light scattering from spin waves in magnetic multilayers with noncollinear spin configurations: theory and experiment," Physical Review B, vol. 75, Article ID 184436, 2007.
[4] P. Manchanda, R. Skomski, P. K. Sahota, M. Franchin, H. Fangohr, and A. Kashyap, "Multiscale micromagnetism of CoPd multilayers," Journal of Applied Physics, vol. 111, Article ID 07C724, 2012.

[5] P. J. Metaxas, J. P. Jamet, A. Mougin et al., "Creep and flow regimes of magnetic domain-wall motion in ultrathin $\mathrm{Pt} / \mathrm{Co} / \mathrm{Pt}$ films with perpendicular anisotropy," Physical Review Letters, vol. 99, no. 21, Article ID 217208, 2007.

[6] H. Salhi, K. Chafai, O. Msieh et al., "Spin-wave excitations in evaporated Co/Pt multilayers," Journal of Superconductivity and Novel Magnetism, vol. 24, no. 5, pp. 1375-1379, 2011.

[7] P. Landeros and D. L. Mills, "Spin waves in periodically perturbed films," Physical Review B, vol. 85, Article ID 054424, 2012.

[8] J. W. Knepper and F. Y. Yang, "Oscillatory interlayer coupling in Co/Pt multilayers with perpendicular anisotropy," Physical Review B, vol. 71, Article ID 224403, 2005.

[9] J. R. Sandercock, "Trends in brillouin scattering: studies of opaque materials, supported films, and central modes," Light Scattering in Solids III, vol. 51, pp. 173-206, 1982.

[10] M. Robinson, Y. Au, J. W. Knepper, F. Y. Yang, and R. Sooryakumar, "Magnetic imaging of layer-by-layer reversal in Co/Pt multilayers with perpendicular anisotropy," Physical Review B, vol. 73, Article ID 224422, 2006.

[11] X. P. Xie, X. W. Zhao, J. W. Knepper, F. Y. Yang, and R. Sooryakumar, "Evolution of magnetic domain reversal with temperature in $\mathrm{Co} / \mathrm{Pt}$ multilayers observed by magnetooptical Kerr imaging," Physical Review B, vol. 76, Article ID 184433, 2007. 

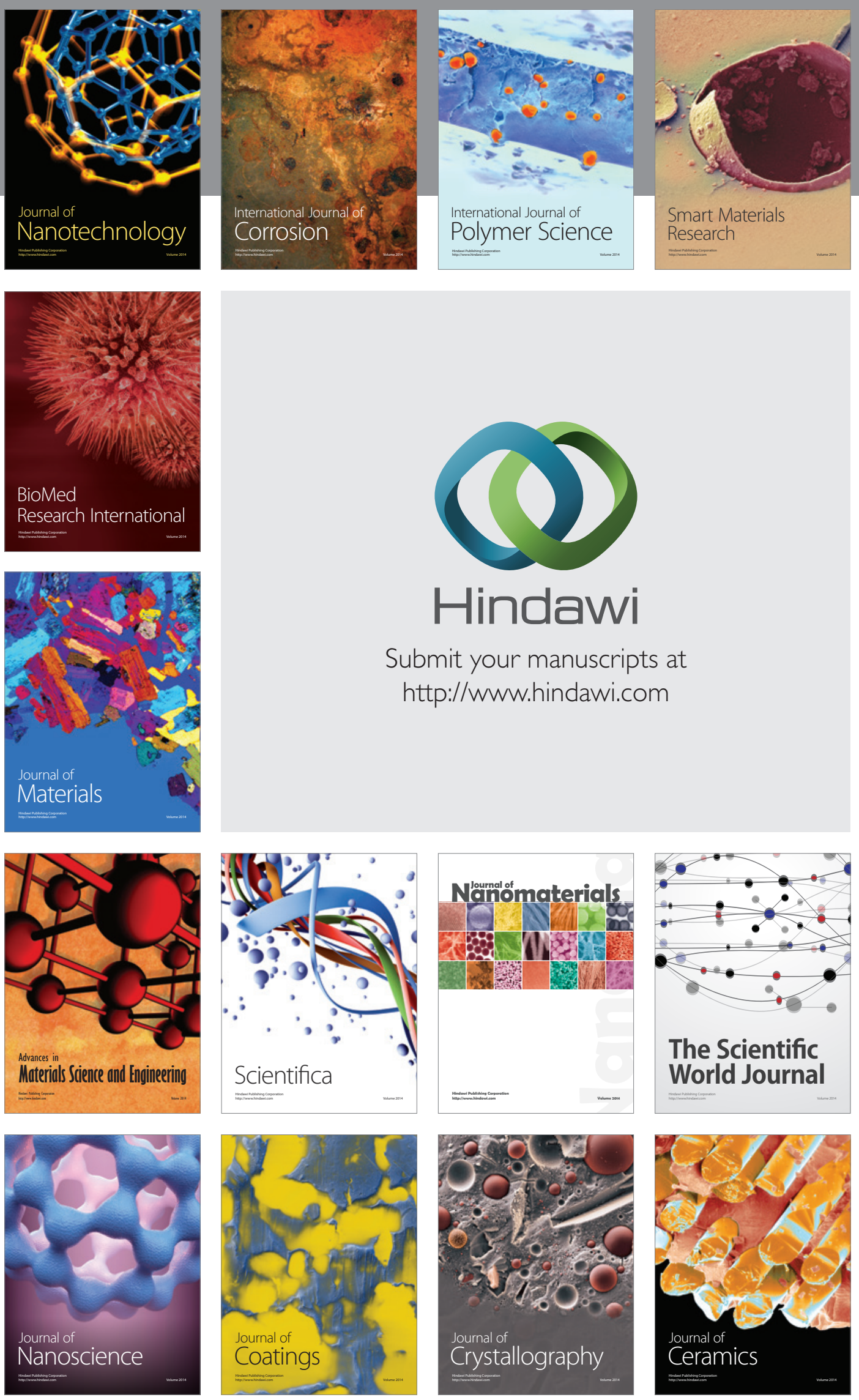

The Scientific World Journal

Submit your manuscripts at

http://www.hindawi.com

\section{World Journal}

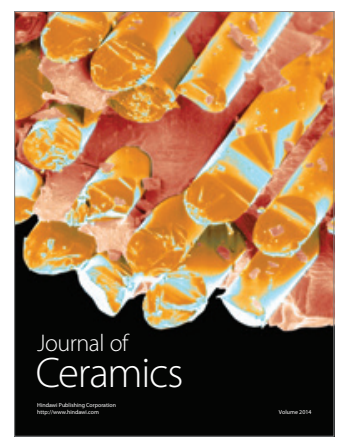

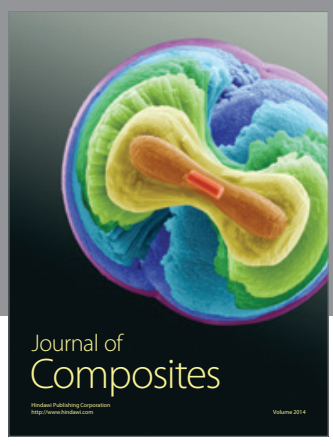
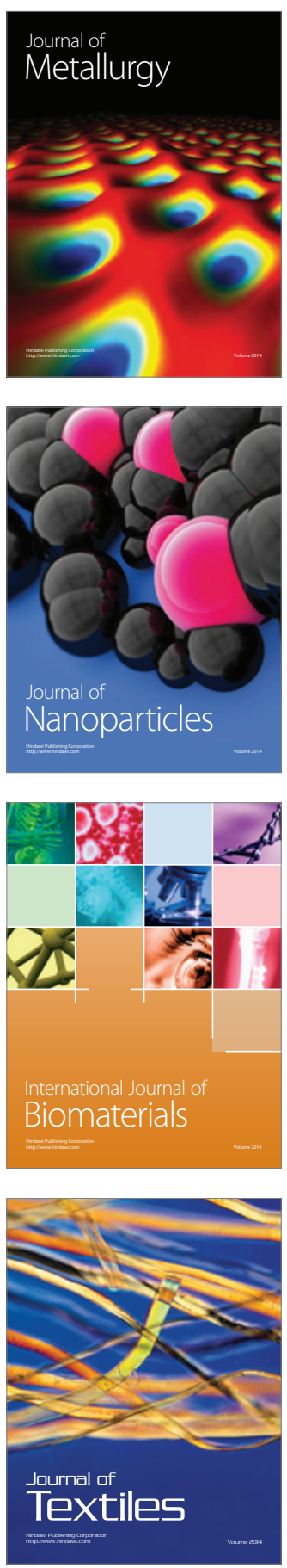